\title{
Locally advanced rectal adenocarcinoma: Are preoperative short and long course radiotherapy truly equivalent?
}

\author{
OFER MARGALIT ${ }^{1,2}$, RONAC MAMTANI ${ }^{3,4}$, YAACOV R. LAWRENCE ${ }^{1,2,5}$, YU-XIAO YANG $^{4,6}$, \\ EREZ N. BARUCH ${ }^{1,2}$, KIM A. REISS $^{3}$, TALIA GOLAN ${ }^{1,2}$, NAAMA HALPERN $^{1,2}$, DAN ADERKA $^{1,2}$, \\ BRUCE GIANTONIO $^{7}$, EINAT SHACHAM-SHMUELI ${ }^{1,2}$ and BEN BOURSI ${ }^{1,2,4}$
}

\begin{abstract}
${ }^{1}$ Department of Oncology, Sheba Medical Center, Tel-Hashomer 52621; ${ }^{2}$ Sackler School of Medicine, Tel-Aviv University, Tel-Aviv 39040, Israel; ${ }^{3}$ Abramson Cancer Center, University of Pennsylvania; ${ }^{4}$ Center for Clinical Epidemiology and

Biostatistics, Perelman School of Medicine at the University of Pennsylvania; ${ }^{5}$ Department of Radiation Oncology, Sidney Kimmel Medical College at Thomas Jefferson University; ${ }^{6}$ Division of Gastroenterology, Department of Medicine, Perelman School of Medicine at the University of Pennsylvania, Philadelphia, PA 19104; ${ }^{7}$ Division of Hematology and Oncology, Department of Medicine, Massachusetts General Hospital, Harvard Medical School, Boston, MA 02114, USA
\end{abstract}

Received September 17, 2018; Accepted March 5, 2019

DOI: $10.3892 / \mathrm{mco} .2019 .1825$

\begin{abstract}
In the neoadjuvant treatment of locally advanced rectal adenocarcinoma, long- and short-course radiotherapy are considered to be of equivalent efficacy based upon several randomized trials. The present study assessed the effect of radiotherapy dose on overall survival. Using the National Cancer Database (2006-2013) 458 individuals with clinical stage II/III rectal adenocarcinoma treated were identified, with either short- (25 Gy) or long- (45 or 50.4 Gy) course neoadjuvant radiotherapy followed by surgery, without neoadjuvant or adjuvant chemotherapy. Multivariate COX regression was employed to evaluate differences in overall survival according to radiotherapy regimen. An association with improved overall survival in individuals treated with long- compared with short-course radiotherapy was demonstrated $(\mathrm{HR}=0.50,0.34-0.73)$. The 30- and 90-day post-surgery mortality rates were higher in the short-course group when compared with the long-course group (12.2 vs. $2.4 \%$; and 18.5 vs. $5.4 \%$, respectively). Following the exclusion of patients that succumbed within 90-days post-surgery, overall survival advantage in the long-course group compared with the short-course group was maintained [hazard ratio $(\mathrm{HR})=0.62,0.39-0.99]$, with a median overall survival of 25.3 months (IQR 16.9-41.6) for the short-course group compared with 43.5 months (IQR 25.6-67.9) for the long-course group. To the best of our knowledge, the present results suggest for the first time that long-course radiotherapy is associated with an improved overall survival compared with short-course radiotherapy in locally advanced rectal
\end{abstract}

Correspondence to: Dr Ben Boursi, Department of Oncology, Sheba Medical Center, Tel-Hashomer 52621, Israel

E-mail: ben.boursi@sheba.health.gov.il

Key words: rectal cancer, radiation, dose, short course, long course adenocarcinoma in the absence of chemotherapy usage. This possible advantage is clinically relevant mainly in patients who cannot tolerate systemic chemotherapy.

\section{Introduction}

Radiotherapy has been shown to improve local control for rectal cancer $(1,2)$. The two commonly used pelvic radiotherapy regimens are either short-course (SC) radiotherapy defined as 25 Gy over 5 consecutive days (3-5) or long-course (LC) chemoradiotherapy defined as 45-54 Gy over 5-6 weeks with concurrent antimetabolite chemotherapy (6-8). The value of adding concomitant chemotherapy has been evaluated only in the long-course radiotherapy, showing improved local control $(9,10)$. Both LC chemoradiotherapy and SC radiotherapy are acceptable options according to current NCCN guidelines.

Two randomized trials that compared LC chemoradiotherapy to SC radiotherapy showed a lack of difference in disease-free survival (DFS) and overall survival (OS) $(11,12$ ). Only one randomized trial compared LC radiotherapy without chemotherapy to SC radiotherapy showing no difference in overall survival (13). However, this trial included patients with upper rectal tumors (ranging from 17 to $28 \%$ in the different trial groups) usually not treated with preoperative radiotherapy, and more importantly did not report clinical stage at diagnosis prior to randomization, making the results difficult to interpret or apply clinically.

In order to assess differences in efficacy between LC and SC radiotherapy in locally advanced rectal adenocarcinoma, we used a US-based nationwide oncology dataset to compare these two regimens without concomitant use of chemotherapy.

\section{Materials and methods}

Data source and patient population. Our cohort was derived from the National Cancer Database (NCDB), a hospital-based 
cancer registry, from 2006 to 2013. The NCDB captures data on $70 \%$ of cancer diagnoses in the United States from $>1,400$ hospitals with cancer programs accredited by the American College of Surgeons' Commission on Cancer and American Cancer Society (14). The cohort included all individuals with clinical stage II (T3-4NOM0) or III (TanyN1-2M0) rectal adenocarcinoma who received neoadjuvant radiotherapy followed by surgery, and did not receive neoadjuvant or adjuvant chemotherapy. The present study was approved by the Institutional Review Board at the University of Pennsylvania (Philadelphia, PA, USA).

Variables definition. The primary exposure of interest was radiotherapy regimen, defined as either SC (25 Gy in 5 Gy fractions) or LC (45 or 50.4 Gy in 1.8 Gy fractions). Covariates included treatment intensity, age, sex, race, patient comorbidities score (Charlson-Deyo comorbidity condition, CDCC) $(15,16)$, and tumor grade. Race and ethnicity were used to create a composite variable categorized as Caucasian, African-American or other/unknown. Tumor grade was defined as well-differentiated, moderately differentiated, poorly differentiated or undifferentiated.

Outcomes definition. The primary outcome was OS, measured from the time of cancer diagnosis until death of any cause or last follow-up.

Statistical analysis. Patients were grouped according to radiotherapy regimen, defined as either SC or LC. Baseline characteristics in each risk group were compared using chi-square test for categorical variables and Student's t-test for continuous variables. OS was measured from date of diagnosis. Difference in OS was compared between the groups, using Cox proportional hazards. The Cox model was adjusted to age, sex, race, CDCC and clinical stage. All statistical analyses were performed using Stata/IC software 13.0 (StataCorp LP, College Station, TX, USA). $\mathrm{P}<0.05$ was considered to indicate a statistically significant difference.

\section{Results}

We identified 458 individuals with stage II or III rectal adenocarcinoma that were treated with radiotherapy followed by surgery, without the use of neither neoadjuvant nor adjuvant chemotherapy. Patients received either SC radiotherapy ( $25 \mathrm{~Gy}, \mathrm{~N}=83$ ) or LC radiotherapy (45/50.4 Gy, N=375). Patient characteristics are presented in Table I. The median follow-up time were 21.7 months (IQR 8.3-37.2) and 41.3 months (IQR 23.3-66.0) for the SC and LC groups, respectively. Patients receiving SC were older than those receiving LC (74 vs. 66 years, respectively, $\mathrm{P}<0.001$ ). All other characteristics were not statistically different between the two groups, although the LC group had numerically more stage III patients compared to the SC group (44.8 vs. $33.7 \%$, respectively). Median interval between diagnosis and surgery was 58 days (IQR 40-87) and 133 days (IQR 111-161) in the SC and LC groups, respectively.

OS according to radiotherapy dose was improved in the LC group compared with the SC group. The unadjusted and adjusted HRs for OS were $0.42(0.29-0.61, \mathrm{P}<0.001)$ and
Table I. Patient characteristics.

\begin{tabular}{|c|c|c|}
\hline \multirow[b]{2}{*}{ Characteristics } & \multicolumn{2}{|c|}{ Radiotherapy protocol } \\
\hline & $\begin{array}{l}\text { Short-course } \\
\qquad(n=83)\end{array}$ & $\begin{array}{l}\text { Long-course } \\
\qquad(n=375)\end{array}$ \\
\hline Age, median (IQR) & $74(65-81)$ & $66(56-77)$ \\
\hline Sex, \% male $(\mathrm{n})$ & $54.2(45)$ & $58.4(219)$ \\
\hline \multicolumn{3}{|l|}{ Race, $\%(n)$} \\
\hline Caucasian & $89.2(74)$ & $85.6(321)$ \\
\hline African-American & $4.8 \quad(4)$ & $8.3(31)$ \\
\hline Other & $6.0 \quad(5)$ & $6.1 \quad(23)$ \\
\hline \multicolumn{3}{|l|}{ CDCC, \% (n) } \\
\hline 0 & $67.5(56)$ & 73.9 (277) \\
\hline 1 & $27.7(23)$ & 18.4 (69) \\
\hline$\geq 2$ & $4.8 \quad(4)$ & 7.7 (29) \\
\hline \multicolumn{3}{|l|}{ Grade, $\%(\mathrm{n})$} \\
\hline Well & $6.0 \quad(5)$ & $7.5 \quad(28)$ \\
\hline Moderate & $59.0(49)$ & $60.8(228)$ \\
\hline Poor & $22.9(19)$ & $14.7 \quad(55)$ \\
\hline Undifferentiated & $1.2 \quad(1)$ & $1.3 \quad(5)$ \\
\hline Other & $10.8(9)$ & $15.7 \quad(59)$ \\
\hline \multicolumn{3}{|l|}{ Clinical stage, $\%$ (n) } \\
\hline II & $66.3(55)$ & $55.2(207)$ \\
\hline III & $33.7(28)$ & $44.8(168)$ \\
\hline
\end{tabular}

CDCC, Charlson-Deyo comorbidity condition; IQR, interquartile range.

$0.50(0.34-0.73, \mathrm{P}<0.001)$, respectively (Fig. 1). This difference in OS was maintained after stratifying patients according to clinical stage. For clinical stage II patients $(n=262)$ the unadjusted and adjusted HRs for OS were 0.50 (0.30-0.82, $\mathrm{P}=0.007)$ and $0.60(0.36-0.99, \mathrm{P}=0.05)$, respectively. For clinical stage III patients $(\mathrm{n}=196)$ the unadjusted and adjusted HRs for overall survival were $0.30(0.18-0.53, \mathrm{P}<0.001)$ and 0.39 (0.22-0.70, $\mathrm{P}=0.002)$, respectively.

Mortality rates post-surgery were higher in the SC group compared with the LC group. Thirty-day mortality rates for the SC and LC were $12.2 \%(\mathrm{n}=10)$ and $2.4 \%(\mathrm{n}=9)$, respectively. Ninety-day mortality rates for the SC and LC were $18.5 \%(\mathrm{n}=15)$ and $5.4 \%(\mathrm{n}=20)$, respectively.

After excluding 35 patients that died within 90-days of surgical resection, OS according to radiotherapy dose was improved in the LC group compared with the SC group. The unadjusted and adjusted HRs for OS were 0.52 (0.33-0.82, $\mathrm{P}=0.005)$ and $0.62(0.39-0.99, \mathrm{P}=0.05)$, respectively (Fig. 2). Median OS was 25.3 months (IQR 16.9-41.6) for the SC group compared to 43.5 months (IQR 25.6-67.9) for the LC group. For clinical stage II patients $(n=241)$ the unadjusted and adjusted HRs for OS were $0.51(0.28-0.94, \mathrm{P}=0.03)$ and $0.60(0.33-1.10, \mathrm{P}=0.1)$, respectively. For clinical stage III patients $(\mathrm{n}=182)$ the unadjusted and adjusted HRs for OS were $0.46(0.23-0.95, \mathrm{P}=0.04)$ and $0.63(0.30-1.34, \mathrm{P}=0.23)$, respectively. 


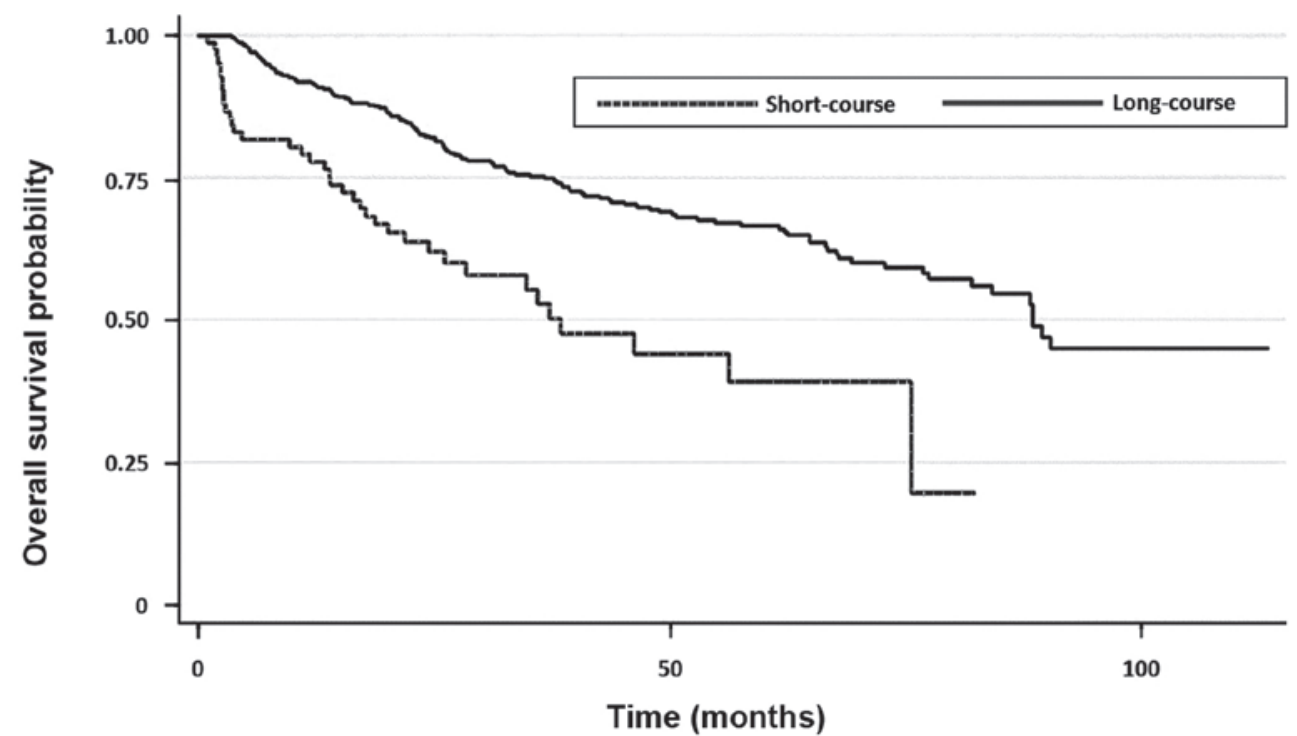

Number at risk

$\begin{array}{llcc}\text { Short-course } & 83 & 12 & 0\end{array}$

$\begin{array}{llll}\text { Long-course } & 375 & 151 & 13\end{array}$

Figure 1. Overall survival among patients with clinical stage II/III rectal adenocarcinoma who received neoadjuvant radiotherapy followed by surgery, according to radiation dose.

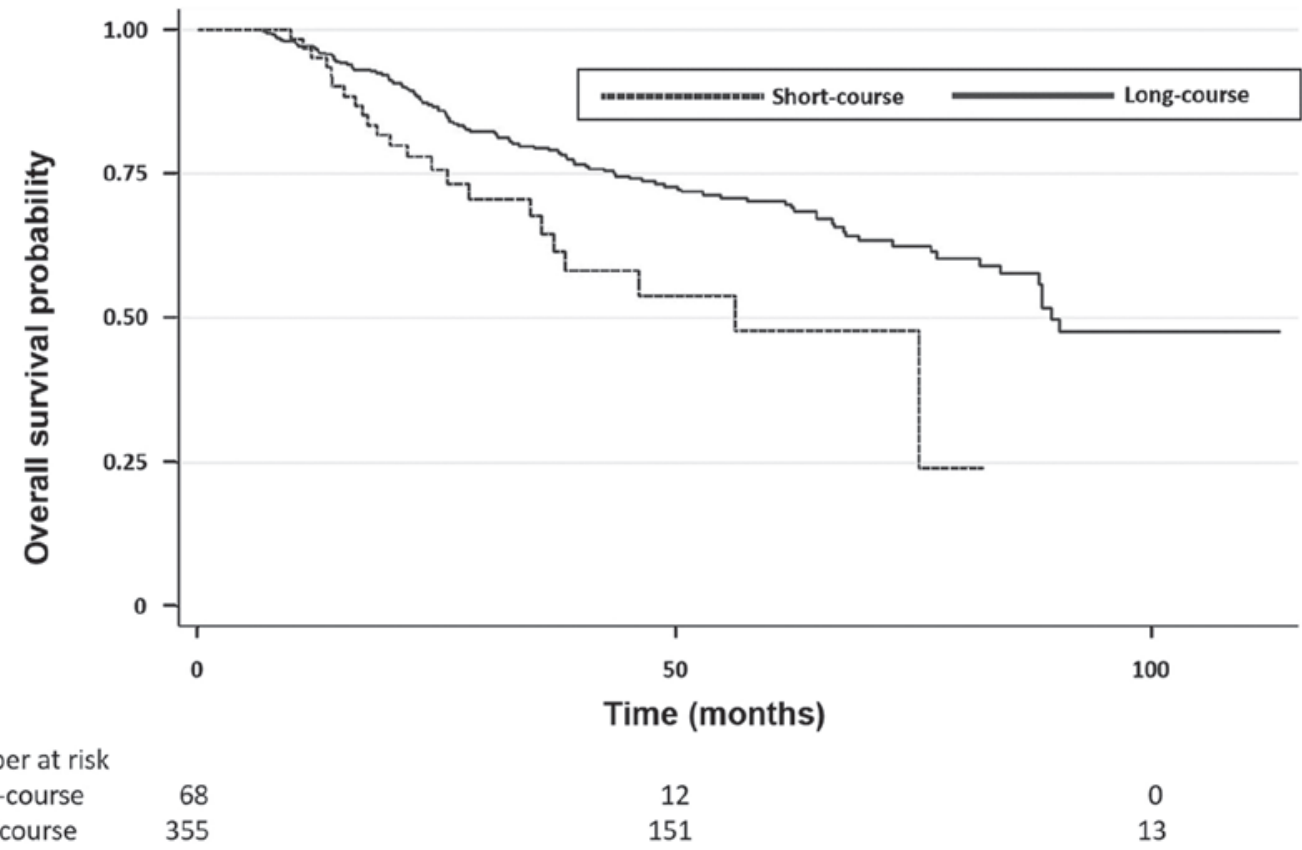

Figure 2. Overall survival among patients with clinical stage II/III rectal adenocarcinoma who received neoadjuvant radiotherapy followed by surgery, following exclusion of early mortality cases, according to radiation dose.

\section{Discussion}

In this study we demonstrated that LC radiotherapy is associated with improved OS compared with SC radiotherapy in patients with locally advanced rectal adenocarcinoma not receiving systemic chemotherapy. In a further analysis we excluded early mortality cases, attributed to patients' poor performance status and comorbidities. Although these cases were more frequent in the SC group, possibly due to older age, the association between LC radiotherapy and improved OS was maintained, and the numerical difference in median OS in favor of the LC group was approximately 18 months.

Preoperative LC chemoradiotherapy was compared with SC radiotherapy in two previous phase III studies, a Polish study and a Trans-Tasman Radiation Oncology Group study $(11,12)$. Both studies showed no difference in OS between the two groups when comparing LC radiotherapy including concomitant chemotherapy of daily infusional 5-fluorouracil to $\mathrm{SC}$ radiotherapy. The association of improved OS with LC radiotherapy in our study may be explained by two factors. 
First, in the Polish study, adjuvant chemotherapy use was more frequent in the SC radiotherapy vs. the LC chemoradiotherapy arm, 46.4 vs. $30.1 \%$, respectively, biasing the results in favor of the SC arm (11). Second, even in the presence of similar rates of adjuvant chemotherapy, as in the Australian study (12), adjuvant chemotherapy by itself may mask the difference in favor of LC over SC radiotherapy. Of note, the biological effective dose (BED) of $\mathrm{LC}$ radiotherapy is significantly higher than that of SC radiotherapy (59.5 vs. 37.5 Gy assuming alpha-beta of 10 and ignoring time factors), in keeping with our findings demonstrating a superiority for LC over SC radiotherapy. The current study suggests that in patients who cannot tolerate systemic chemotherapy, LC should be preferred over SC radiotherapy.

Strengths of our study include the large size of the cohort used for analysis, the detailed available information for clinical staging, the ability to clearly define patients who received preoperative radiotherapy followed by surgery, and the adjustments performed for the common confounders (i.e., age, sex, race, comorbidities and clinical stage). All these have enabled the direct comparison between LC and SC radiotherapy, focusing on the effect of radiation dose and intensity on OS.

This study had several important limitations. First, our dataset also lacks cancer recurrence and cancer-specific survival data. However, NCDB includes OS data, which is a more clinically relevant parameter. Second, other confounding factors affecting OS cannot be ruled out. Third, since the time from diagnosis to surgery was shorter in the SC compared with the LC group, there was a small component of an immortal time bias. However, this difference is negligible (approximately 14\%) compared with the difference in median overall survival. Fourth, details of radiation technique are lacking. Fifth, NCDB lacks data regarding toxicity and side effects in patients that received LC or SC radiotherapy. Finally, the reasons for omitting neoadjuvant/adjuvant chemotherapy in both LC and SC groups cannot be fully elucidated, which may potentially bias overall survival. However, we adjusted our analysis for the main factors affecting treatment decision, i.e., age, comorbidities and clinical stage.

In summary, our findings suggest that LC radiotherapy is associated with an improved OS compared with SC radiotherapy in locally advanced rectal adenocarcinoma. To the best our knowledge, this is the first report in the literature suggesting an advantage for LC radiotherapy. This possible advantage is clinically relevant mainly in patients who cannot tolerate adjuvant chemotherapy, and should therefore be taken into account in the decision-making process in this setting.

\section{Acknowledgements}

Not applicable.

\section{Funding}

No funding was received.

\section{Availability of data and materials}

The datasets generated and/or analyzed during the current study are not publicly available due to the University's licensing agreement with NCDB which prohibits the distribution of this data to any individual not explicitly listed in the originally agreed proposal.

\section{Authors' contributions}

OM, YRL, YXY, ENB, KAR, TG, NH, DA, BG and ESS contributed to the study design, data analysis and drafting the manuscript. RM contributed to the acquisition of data, statistical analysis and drafting the manuscript. BB contributed to the study design, acquisition of data, statistical analysis and drafting the manuscript. All authors read and approved the final manuscript.

\section{Ethics approval and consent to participate}

The present study was approved by the Institutional Review Board at the University of Pennsylvania (Philadelphia, PA, USA).

\section{Patient consent for publication}

Not applicable.

\section{Competing interests}

All authors declare that they have no conflict of interest.

\section{References}

1. Cammà $\mathrm{C}$, Giunta $\mathrm{M}$, Fiorica $\mathrm{F}$, Pagliaro L, Craxì $\mathrm{A}$ and Cottone M: Preoperative radiotherapy for resectable rectal cancer: A meta-analysis. JAMA 284: 1008-1015, 2000.

2. Colorectal Cancer Collaborative Group: Adjuvant radiotherapy for rectal cancer: A systematic overview of 8,507 patients from 22 randomised trials. Lancet 358: 1291-1304, 2001.

3. Birgisson H, Påhlman L, Gunnarsson U and Glimelius B; Swedish Rectal Cancer Trial Group: Adverse effects of preoperative radiation therapy for rectal cancer: Long-term follow-up of the Swedish Rectal Cancer Trial. J Clin Oncol 23: 8697-8705, 2005.

4. Marijnen CA, Kapiteijn E, van de Velde CJ, Martijn H, Steup WH, Wiggers T, Kranenbarg EK and Leer JW; Cooperative Investigators of the Dutch Colorectal Cancer Group: Acute side effects and complications after short-term preoperative radiotherapy combined with total mesorectal excision in primary rectal cancer: Report of a multicenter randomized trial. J Clin Oncol 20: 817-825, 2002.

5. Sebag-Montefiore D, Stephens RJ, Steele R, Monson J, Grieve R, Khanna S, Quirke P, Couture J, de Metz C, Myint AS, et al: Preoperative radiotherapy versus selective postoperative chemoradiotherapy in patients with rectal cancer (MRC CR07 and NCIC-CTG C016): A multicentre, randomised trial. Lancet 373: 811-820, 2009.

6. Park JH, Yoon SM, Yu CS, Kim JH, Kim TW and Kim JC: Randomized phase 3 trial comparing preoperative and postoperative chemoradiotherapy with capecitabine for locally advanced rectal cancer. Cancer 117: 3703-3712, 2011.

7. Roh MS, Colangelo LH, O'Connell MJ, Yothers G, Deutsch M, Allegra CJ, Kahlenberg MS, Baez-Diaz L, Ursiny CS, Petrelli NJ, et al: Preoperative multimodality therapy improves disease-free survival in patients with carcinoma of the rectum: NSABP R-03. J Clin Oncol 27: 5124-5130, 2009.

8. Sauer R, Liersch T, Merkel S, Fietkau R, Hohenberger W, Hess C, Becker H, Raab HR, Villanueva MT, Witzigmann H, et al: Preoperative versus postoperative chemoradiotherapy for locally advanced rectal cancer: Results of the German CAO/ARO/AIO-94 randomized phase III trial after a median follow-up of 11 years. J Clin Oncol 30: 1926-1933, 2012. 
9. Bosset JF, Collette L, Calais G, Mineur L, Maingon P, Radosevic-Jelic L, Daban A, Bardet E, Beny A and Ollier JC; EORTC Radiotherapy Group Trial 22921: Chemotherapy with preoperative radiotherapy in rectal cancer. N Engl J Med 355: 1114-1123, 2006.

10. McCarthy K, Pearson K, Fulton R and Hewitt J: Pre-operative chemoradiation for non-metastatic locally advanced rectal cancer. Cochrane Database Syst Rev 12: CD008368, 2012.

11. Bujko K, Nowacki MP, Nasierowska-Guttmejer A, Michalski W, Bebenek M and Kryj M: Long-term results of a randomized trial comparing preoperative short-course radiotherapy with preoperative conventionally fractionated chemoradiation for rectal cancer. Br J Surg 93: 1215-1223, 2006.

12. Ngan SY, Burmeister B, Fisher RJ, Solomon $M$, Goldstein D, Joseph D, Ackland SP, Schache D, McClure B, McLachlan SA, et al: Randomized trial of short-course radiotherapy versus long-course chemoradiation comparing rates of local recurrence in patients with $\mathrm{T} 3$ rectal cancer: Trans-Tasman Radiation Oncology Group trial 01.04. J Clin Oncol 30: 3827-3833, 2012.
13. Erlandsson J, Holm T, Pettersson D, Berglund A, Cedermark B Radu C, Johansson H, Machado M, Hjern F, Hallböök O, et al: Optimal fractionation of preoperative radiotherapy and timing to surgery for rectal cancer (Stockholm III): A multicentre, randomised, non-blinded, phase 3, non-inferiority trial. Lancet Oncol 18: 336-346, 2017.

14. Winchester DP, Stewart AK, Bura C and Jones RS: The National Cancer Data Base: A clinical surveillance and quality improvement tool. J Surg Oncol 85: 1-3, 2004.

15. Charlson ME, Pompei P, Ales KL and MacKenzie CR: A new method of classifying prognostic comorbidity in longitudinal studies: Development and validation. J Chronic Dis 40: 373-383, 1987.

16. Deyo RA, Cherkin DC and Ciol MA: Adapting a clinical comorbidity index for use with ICD-9-CM administrative databases. J Clin Epidemiol 45: 613-619, 1992. 